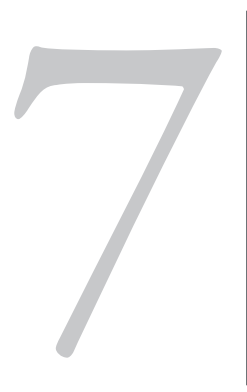

\title{
EL MODELO DUAL-INCREMENTAL DE REGIONALIZACIÓN EN CHILE: INTENDENTES ELECTOS Y GOBERNADORES DELEGADOS
}

\author{
DUAL-INCREMENTAL MODEL OF \\ REGIONALISATION IN CHILE: \\ ELECTED INTENDENTES AND \\ DELAGATED GOVERNORS
}

Esteban Valenzuela Van Treek ${ }^{2}$

Recibido: 30/01/2015

Aceptado: 13/07/2015

\section{R E S U M E N}

El artículo analiza la pugna por el moderado proceso de descentralización regional que impulsa el Gobierno chileno, replicando lo que se puede llamar la experiencia francesa de los 1980s: La Nueva Mayoría gobernante (partidos y ministros/ agencias públicas claves) articuló un modelo dual de poder para las regiones, permitiendo la elección de Intendentes junto a una delegación central fuerte a través de gobernadores delegados que coordinarán la nutrida red de agencias estatales desconcentradas. Dicho proceso se complementa con un acotado proceso de traspaso de competencias que tomará una década tras pilotajes, donde se incluyeron sólo algunos de los servicios con las competencias esenciales a los entes regionales de los países de la OECD. Se complementa con una propuesta de aumento de los pequeños impuestos a actividades vinculadas a recursos naturales y puertos. Todo ello explica tanto el avance de los sectores reformistas/ centralista como la con la racionalidad centralista/iluminista que predomina en la elite chilena. La ley de elección de intendentes con fuerte delegación central y de traspaso de competencias limitado se contrasta con el enfoque más autonomista de las propuestas de la Comisión Asesora Presidencial para la Descentralización y el Desarrollo Regional que reunió a 33 expertos en el año 2014.

\section{PA L A R A S C L A V E S}

Regionalización, descentralización, Gobierno Bachelet, intendentes, competencias.

1. FONDECYT "Movimientos Regionalistas y Transformaciones políticas en Chile 2011-2016: Entre la cooptación y el poder autónomo" №1150684.

2. Departamento de Ciencia Política y Relaciones Internacionales de la Universidad Alberto Hurtado. Correo electrónico: evalenzuelavt@gmail.com 


\section{A B S T R A C T}

The article analyzes the moderate regional decentralization process promoted by the Chilean government, replicating what can be called the French experience of the 1980s: the ruling coalition Nueva Mayoria (parties and ministers / key public agencies) articulated a dual model of power for the regions, allowing the election of intendentes (Chilean chief of the region) with strong central delegation through delegated governors who will coordinate the dense network of desconcentrated state agencies. This process is complemented by a limited devolution process that will take a decade after piling, which included only some of the core services for the new autonomous regional government, far away with the competencies of the regional authorities of the countries of the OECD. It is complemented by a proposal to increase taxes on small activities related to natural resources and ports. This explains the progress of the reformist sectors, but the strong power of old centralist guard with a centralist / Enlightenment rationality that dominates the Chilean elite. The election of intendentes law with strong central delegation and limited transfer of powers is contrasted with the more autonomous approach proposed by the Presidential Advisory Commission for Decentralization and Regional Development, which brought together 33 experts in 2014.

\section{KE Y W O R D S}

Regionalization, decentralization, Bachelet government, intendentes, competencies.

\section{INTRODUCCIÓN: EL PROGRAMA REFORMISTA DE B ACHELET, LA DEMANDA REGIONALISTA Y LOS TRES PILARES DE TODA DESCENTRA- L I Z A C I Ó N}

El punto de vista del artículo es valorar la descentralización como fin democrático (dispersión del poder) y como medio para el desarrollo cuando ella se produce con seriedad, sin duplicidades ni despilfarro, con fondos de convergencia, evaluación de impactos y el adecuado control social e institucional como lo demandan los movimientos sociales territoriales (Delamaza, Cunill y Joignant 2012, Valenzuela y Penaglia 2014). Chile ha sido fuerte y exitoso en tener estatalidad en todo el territorio para cumplir con servicios sociales básicos convergentes y homogéneos, pero retrasado en autonomía administrativa y fiscal (Rosales 2012, Brosio y Jiménez 2012) que dinamice políticas de fomento del capital social, medio ambiente, transporte y competitividad.

Chile entró en el año 2010 en un período de protestas por educación pública 
y socio territoriales que empujaron el llamado nuevo ciclo de reformas que hizo retornar al poder a Michelle Bachelet el año 2014 con una nueva coalición de centro-izquierda llamada Nueva Mayoría (incorporó a comunistas y otros grupos de izquierda ajenos a la antigua Concertación), la que adoptó un discurso transformador incluyendo la elección de los intendentes regionales y el traspaso de poderes a regiones y municipios, rompiendo discursivamente el tradicional centralismo de la elite chilena (Valenzuela 2015). Para cumplir su programa y consensuar las propuestas, Bachelet nominó por seis meses en el año 2014 una Comisión Asesora Presidencial para la Descentralización y el Desarrollo Regional, la que le propuso tres medidas esenciales: elección de intendentes, traspaso de competencias y ley de rentas regionales. Además, dicha Comisión realizó quince cabildos masivos en todas las regiones, donde se corroboró el resentimiento por los pocos recursos (sobre todo en el norte minero), la falta de programas en transporte y medio ambiente (Coquimbo, Valparaíso, centro-sur) y la débil autonomía para resolver conflictos (Araucanía) y generar mega proyectos y desarrollo productivo (O’Higgins y Bío-Bío, entre otras).

Al recibir el 7 de octubre el Informe de dicha Comisión, la Presidenta anunció la reforma de elección de intendentes, el proceso de traspaso de competencias (ambos ingresados a trámite en el Senado de la República en enero del 2015) y para el segundo semestre del 2015 la ley de financiamiento de los gobiernos regionales y responsabilidad fiscal de los mismos ${ }^{3}$.

Como indican la escolástica sobre descentralización (Faletti, 2010, Navarrete e Higueras, 2014, Rosales, 2012, Valenzuela, 2014) existe un ABC que es combinar lo político (elección de autoridades con poderes), lo administrativo (traspaso de competencias claras) y económicas (tributos propios y/o asegurados por ley). Desde dicho esquema de comparación, se observan las diferencias de los proyectos y modelo consensuado por los partidos de la Nueva Mayoría con la propuesta articulada por la Comisión Asesora Presidencial para la Descentralización y el Desarrollo Regional ya que esta proponía en lo político no sólo elegir el Intendente sino fortalecer las potestades constitucionales del gobierno regional autónomo, incluyendo un modelo de mediación en caso de disputa por competencias. Lo que llamaron "reforma constitucional para la descentralización" como primera medida esencial ${ }^{4}$. También se consideraba avanzar en lo municipal, ante lo cual el Gobierno lo ha diferenciado en su anuncio de un programa de nueva agenda municipal. Finalmente, la Comisión propuso como esencial los temas de participación y eso ha quedado también postergado para otras agendas.

3. Compromiso que refrendó en su cuenta presidencial del 21 de mayo del 2015 en la sede del Congreso en Valparaiso.

4. Informe Comisión Asesora Presidencial para la Descentralización y la Regionalización, Octubre 2014. p 23. 
No obstante, el gobierno ha sido coherente con esta idea de los tres factores claves, al concentrarse en estos tres proyectos en función de lo político, administrativo y lo fiscal para junio del 2015 que logran poner en marcha un proceso político-legislativo que puede poner a Chile en una fase descentralizadora- a pesar de la desmunicipalización de la educación-, a contra corriente de los procesos de re-centralización que vive el Continente en cuanto a los procesos de concentración de la riqueza, la producción y los ingresos así como también la asignación y ejecución sectorial y centralizada por parte del Estado (Cravacuore 2014 y Finot, 2007).

El tercer aspecto que es la descentralización fiscal no arranca en el segundo año de Gobierno, ya que se posterga para el anuncio de ley de financiamiento de los gobiernos regionales (sin fechas claras de operacionalización) y con una versión acotada de la idea de Fondo de Convergencia Regional (en el programa de Bachelet) por la vía de agrupar recursos especialmente para las zonas extremas. Lo señala con claridad Camilo Vial del ICHEM: "Proporcionalmente las regiones no tendrán más recursos que el gobierno central en 2015. Por el contrario, se mantendría la tendencia: de aprobarse, el presupuesto de los GORE equivaldrá sólo al 3,06\% del que proyecta el gobierno central. El promedio de los últimos 10 años ha sido de 2,92\%. En otras palabras, el presupuesto regional aumentaría en la misma medida en que se incrementaría el del país" ${ }^{5}$.

\section{LOSECOS DEL PODER DUALTERRITORIAL} DE FRANCIA EN EL ESQUEMA DEL GOBIERNO

Chile adopta claramente un modelo que hace cohabitar entes subnacionales con poder acotado junto a una fuerte presencia de agencias regionales que deben coordinar sus planes en un entramado fuerte. Por razones históricas Chile ha tendido también una elite tanto de derecha e izquierda que ha avalado el modelo institucional de gobierno central fuerte con oficinas desconcentradas en los territorios, municipios con moderado poder y delegación regional con baja autonomía política, económica y administrativa (Valenzuela 1999). En el programa de los partidos por el NO y luego Concertación, el cambio de régimen político (de lo presidencial a lo semi parlamentario y del centralismo a la descentralización), no tuvo espacio programático y se tendió a administrar el modelo centralista presidencialista, no obstante con sucesivas reformas que fueron empoderando y democratizando el nivel subnacional. La Concertación de Partidos por la Democracia realizó en el gobierno de 
Patricio Aylwin (1990-93) el proceso de democratización de los municipios (incluyendo aquellos que en la democracia pre 73 eran designados, como Santiago, Valparaíso, Viña del Mar, Concepción, entre otros) y la creación de consejos regiones (en vez de entes corporativos gremiales heredados del régimen militar) electos de manera indirecta a nivel provincial por asambleas de concejales municipales. A dichos gobiernos regionales se les dotó también de facultades reglamentarias y de competencias generales en desarrollo.

Luego en el gobierno de Frei Ruiz-Tagle no se cumplió la promesa de elegir de manera directa a los consejeros regionales, pero si se avanzó en transferir a los gobiernos regionales y al CORE mayor poder de negociación con los ministerios centrales por la vía de ampliar el uso de inversiones pactadas por la vía de Inversión sectorial a asignación regional (ISAR) y Convenios de Programación de carácter multianual.

El Gobierno de Ricardo Lagos (2000-2005), al fragor del empate electoral con el candidato de la derecha, se abrió a una postura de mayor reformismo e hizo suya la mayoría de las propuestas de un programa de 20 expertos en descentralización agrupados por la Fundación Friedrich Ebert vinculada a la socialdemocracia alemana (Martelli y Valenzuela 1999). En dicha propuesta ya se tuvo el modelo francés como manera de lograr avanzar la descentralización, al proponerse la elección de un Presidente regional que liderara el CORE, cohabitando con el Intendente electo. A lo anterior se sumaba la elección de COREs, áreas metropolitanas, traspaso de servicios y ley de financiamiento. No hubo avances en dicho gobierno.

La Presidenta Bachelet en su primero gobierno (2006-2009) logró fortalecer las capacidades de los gobiernos regionales y potestades (entre ellas, pedir traspaso de competencias), crear las dos nuevas regiones de Los Ríos y Arica-Parinacota y aprobar la reforma constitucional para la elección directa de los consejeros regionales, que implementó el Gobierno de Sebastián Piñera (2010-2013).

El Gobierno de Piñera preparó un proyecto misceláneo de fortalecimiento de los gobiernos regionales con mayor detalle de fórmulas para traspaso de competencias, pero sin plazos perentorios ni servicios explícitos. No obstante, dicho proyecto fue valorado por el segundo gobierno de Bachelet y sobre el mismo construyó las indicaciones para el traspaso de competencias y otras medidas como la manera de nombrar las regiones. En la metarelato se observa la voluntad de incrementalidad histórica en un proceso gradual a la chilena: 
El proyecto de ley que modifica la ley № 19.175, Orgánica Constitucional sobre Gobierno y Administración Regional, profundizando la regionalización del país, aprobado en primer trámite constitucional por el H. Senado el 8 de enero de 2014, dispone, entre diversas otras materias, un procedimiento de transferencia de competencias y dispone la obligatoriedad de los convenios de programación encontrándose ausente los dos temas restantes. Sin perjuicio de que se conserva gran parte de lo planteado en el Mensaje original y los valiosos aportes del H. Senado, mediante la presente indicación sustitutiva se pretende perfeccionar y profundizar la regulación dispuesta para el mecanismo de transferencia de funciones y atribuciones y, a la vez, añadir, entre otras materias, la eliminación de la identificación de las regiones mediante números y la administración de áreas metropolitanas. En consecuencia, es voluntad de mi Gobierno completar y finalizar íntegramente, a través de la presente iniciativa, la regulación legal de la reforma constitucional sobre materias regionales aprobada durante el primer Gobierno que presidí ${ }^{6}$.

La Presidenta Bachelet generó altas expectativas con su agenda descentralizadora que incluyó la Comisión Asesora Presidencial para la Descentralización y el Desarrollo Regional del año 2014 que propuso la elección de intendentes, traspaso de competencias relevantes, ley de rentas regionales, apoyo a las capacidades regionales en capital humano y modelos de participación relevantes incluyendo revocatoria de cargos ${ }^{7}$. De esta manera se hizo cargo del ciclo de protestas demandando mayor poder territorial (Penaglia y Valenzuela 2014) que tuvo en el gobierno de Piñera casos emblemáticos (Calama, Aysén y Magallanes) sumado a la década y media de protestas mapuches (Marimán 2012).

El proyecto enviado al Congreso en diciembre del año 2014 propone que el Intendente sea el electo y a su vez sea quien preside el Consejo Regional (como lo propuso la Comisión Presidencial), pero no aceptó definir Chile como Estado descentralizado ni acotar la delegación central a los gobernadores provinciales, generando la fuerte figura del gobernador regional. Mientras la Comisión proponía un delegado nacional en todas las provincias (con la idea de que coordinara servicios nacionales el delegado de la gobernación provincial cabecera de la capital regional), el Ejecutivo decidió como fórmula

6. Formula indicación sustitutiva al proyecto de ley que introduce modificaciones a la ley $n^{\circ} 19.175$, orgánica constitucional sobre gobierno y administración regional, profundizando la regionalización del país (Boletín $\mathrm{N}^{\circ}$ 7963-06). 16 de enero 2016.

7. Tal cual se puede ver la exposición de los resultados de la Comisión en el Senado. Disponible en: http:// www.senado.cl/informe-final-de-comision-de-descentralizacion-fue-entregado-en-el-congreso/prontus_ senado/2014-10-07/112343.html 
de compromiso al interior de la Nueva Mayoría crear una figura fuerte: el gobernador regional. Varias autoridades advirtieron excesos "federalistas" en privado en el contexto del Comité Político, especialmente de los presidentes del PS y de la DC, colocando dudas con la fecha de elección de intendentes el 2017: “La semana pasada, los presidentes del PS y la DC, Osvaldo Andrade e Ignacio Walker, plantearon en privado las dificultades de hacer las elecciones ese año, debido a que aún no está bien definido el mecanismo y tampoco hay certeza de que alcancen los trámites legislativos. Ambos propusieron como fecha más realista el 2020 o 2021. En esa oportunidad, la postura de los parlamentarios fue resistida por el PC, MAS y el PPD"8.

La propuesta de elección de intendentes por parte de la Presidenta en su campaña había generado altas expectativas porque acercaba a Chile más a los países unitarios fuertemente descentralizados (España y Colombia). No obstante sus equipos político técnicos fueron morigerando al hablar sólo de elección de ejecutivo regional y omitir toda referencia a la ley de rentas regionales. En estricto rigor, el Gobierno ha puesto el caso chileno en una mayor continuidad con el modelo francés iniciado por Francois Miterrand en el más centralista país de la OCDE, Francia, quien en los 1ños 1980s logró la reforma para elegir a los Presidentes regionales, pero con la permanencia de un fuerte prefecto en los territorios poblados de agencias estatales de diferente tipo.

La idea de abandonar la referencia al Estado unitario- por una definición de Estado "único y regionalizado" no ponía en duda la unidad del país. De hecho, la propia Francia - regularmente presentado como el país unitario por excelencia- no es más definido por la Constitución como unitario sino como República indivisible (Debbasch 1988). El constitucionalista francés, Benoît Delooz recuerda que la mención tradicional de la unidad de la República despareció en 1958, al menos que la noción haya sido disuelta en la de indivisibilidad. Chile en cambio ha temido modificar su carácter unitario a pesar lo caminado por la propia Francia que junto al fuerte gobierno central acepta niveles de autonomía en sus territorios de ultramar (la Polinesia) y cada vez más fuertes gobiernos regionales, metropolitanos y otras formas de intercomunalidad.

8. Ver "Gobierno anuncia elección de intendentes en 2017 pero se abre a retrasar comicios" (2014). Disponible en: http://www.lasegunda.com/Noticias/Politica/2014/12/984218/gobierno-anuncia-eleccion-de-intendentes-en-2017pero-se-abre-a-retrasar-comicios 
La referencia al carácter unitario, desde la Revolución francesa es demasiado asociado a la uniformidad de las instituciones que era dogmáticamente considerada como inherente al principio de igualdad. Sin embargo, la realidad es la diversidad de las situaciones y se debe considerar, quizás, al lado de un marco común, la posibilidad de tener entes territoriales con estatutos particulares. Por otro lado, ya existen fórmulas de cooperación, asociación y/o fusión interinstitucional ${ }^{9}$ cuya evolución puede cambiar el mapa de la administración territorial del país". (Delooz, 2014, p. 36-37).

La descentralización comenzó en 1982 estableciendo los derechos y libertades de las comunas, departamentos y regiones. Esta es la primera y más importante ley de descentralización de la historia de la República francesa. Fue clave, entonces, la supresión de la tutela administrativa, eliminando el rol del control que el Prefecto delegado central tuvo históricamente sobre los entes subnacionales. La transformación de las regiones en colectividades territoriales tuvo el mismo poder autónomo que los municipios, como el paso a seguir en materia de descentralización.

El proceso de traspaso fue de inmediato, con varias leyes que completaron estas medidas. Las primeras transferencias de competencias fueron realizadas por dos leyes del 1983. La ley de 1984 definió el estatuto de los agentes empleados por las colectividades territoriales: es la creación de la función pública territorial. Una ley de 1987 reforzó el poder de nominación de las colectividades. Una ley de 1992 organizó la cooperación entre las colectividades y el desarrollo de vínculos entre ellas, lo que se llama oficialmente como la "intercomunalidad".

Se vivieron procesos orientados a profundizar en materia de descentralización, la ley de 28 de marzo de 2003 reactivó el proceso de fortalecimiento de las comunidades subnacionales. A través de la modificación de la Constitución, la ley añade varios puntos en su carta magna. Establece que "la organización de la República es descentralizada" (Artículo Nº1). Reafirmando que la región es una colectividad territorial y el principio de la autonomía financiera de las colectividades (Artículo $\mathrm{N}^{\mathrm{0}} 72$ ). Por fin crea la posibilidad de referéndums locales que tienen un poder de decisión (Artículo N²7). De esta manera, la ley de 2004 organizó otras transferencias de poderes en ámbitos económicos, políticos y administrativos. 
Sin embargo, la crisis económica de Europa desde el año 2009, ha generado déficits fiscales que han obligado a los gobiernos a simplificar niveles de gobierno, evitar duplicidades y en el caso de Francia a un proceso a racionalización y austeridad de los niveles subnacionales que se tradujo en una disminución del número de regiones:

Uno de los objetivos del histórico cambio consiste en ahorrar entre 12.000 y 25.000 millones de euros en los próximos años. Las actuales 22 regiones se fusionan entre ellas. Quedarán 13. El principal argumento del presidente François Hollande y de su Gobierno para semejante revolución consiste en lograr una gestión pública más ágil y menos costosa (hoy se lleva el 55\% del PIB) repartida hoy entre la Administración central, las 36.700 comunas o ayuntamientos (el país con más entes locales del mundo), las 13.400 mancomunidades, los 96 departamentos o provincias con sus respectivos consejos generales o asambleas (4.058 cargos electos con sueldo) y las 22 regiones con sus consejos regionales (1.757 cargos). Las competencias, en ocasiones, están duplicadas ${ }^{10}$.

3. LAS PUGNAS CLAVES ENTRE DETRACTORES A NTI-FEDERALISTAS Y PARTIDARIOS DE MAYOR PODER REGIONAL

La oposición ha tenido dos segmentos diferenciados respecto a este proceso de reforma. Por una parte, la UDI, el Instituto Libertad y Desarrollo, mantienen su postura municipalista que no gusta de traspaso de poderes mayores al nivel regional porque la desconocen como esfera de deliberación. Para la derecha los municipios son el espacio que, por ser más cercano a la ciudadanía, debería ser el más apto para recibir poder y autogestionarse en materias económicas, políticas y administrativas. Los municipios ya cuentan con una madurez y por tanto los esfuerzos en descentralizar el país deben concentrarse en las localidades (Horst, 2007).

10. Uno de los objetivos del histórico cambio consiste en ahorrar entre 12.000 y 25.000 millones de euros en los próximos años. Las actuales 22 regiones se fusionan entre ellas. Quedarán 13. 
A su vez, los medios editoriales de El Mercurio y La Tercera, sistemáticamente han criticado que esta reforma puede debilitar el Estado unitario, acentuar procesos de irresponsabilidad fiscal subnacional y hacer declinar políticas regionales en un pseudo federalismo sin control. En diversas notas periodísticas se han profundizado el debate en torno a la fecha para la elección de los intendentes, dando espacio a quienes pretenden retrasar la elección para el 2017 o $2020^{11}$.

La otra vertiente ha sido favorable a la reforma y ha criticado sus debilidades. La propuesta de RN al Gobierno converge en la idea de Estado unitario descentralizado, no le gusta propuestas participativas de la Comisión Asesora Presidencial para la Descentralización y el Desarrollo Regional como aquella de revocatorio popular de mandatos, pero sí apoya las rentas regionales y un traspaso de poderes/competencias/servicios al Intendente electo, evitando duplicidad con el gobernador regional: "Servicios que queden entregados al Intendente Regional deben desaparecer de la competencia del Gobernador Regional para evitar la duplicidad de funciones y un derroche de recursos públicos"12.

Los más activos segmentos de centro derecha de la Comisión presidencial para la Descentralización fueron su vicepresidente, Heinrich Von Baer (ex rector de la U. de la Frontera-Temuco y presidente del Consejo Nacional para la Descentralización y Regionalización, CONADERE) y el abogado constitucionalista Juan Andrés Varas, ex decano de derecho de la U. Austral-Valdivia e Intendente de la Región de Los Ríos en el gobierno de Sebastián Piñera (2010-13). El foco de las críticas fue:

\section{A. LA POSTERGACIÓN DE LA FECHA DE ELECCIÓN DEL INTEN- DENTE PARA LAS PRESIDENCIALES DE FINES DEL 2017 EN VEZ DE LA PROPUESTA DE HACERLA JUNTO A LAS MUNICIPALES EL 2016.}

Una opinión distinta plantea el vicepresidente de la comisión, Heinrich von Baer, al establecer que le parece "preocupante que finalmente se proponga

11. Ver nota de La Tercera "Gobierno envía proyecto para elección de intendentes y abre debate por fecha de comicios" (2014). Disponible en: http://www.latercera.com/noticia/politica/2014/12/674-610805-9-gobierno-enviaproyecto-para-eleccion-de-intendentes-y-abre-debate-por-fecha-de.shtml

Ver nota de El Mercurio "Presidenta Michelle Bachelet firma indicaciones a proyecto de descentralización del país" (2015). Disponible en: http://www.emol.com/noticias/nacional/2015/01/16/699525/presidenta-bachelet-firmaindicaciones-a-proyecto-de-ley-de-descentralizacion-del-pais.html

12. Ver nota de El País "Francia revoluciona en dos meses su mapa regional" (2014). Disponible en: http:// internacional.elpais.com/internacional/2014/07/31/actualidad/1406797786_782418.html 
realizar esta primera elección de intendentes recién en 2017, simultánea a las elecciones presidenciales y parlamentarias, y no en octubre de 2016, paralela a las municipales, como propuso la comisión", con el objetivo de que no quede "opacada" por la alta visibilidad de la elección presidencial. Von Baer respaldó el planteamiento de la Comisión Asesora Presidencial para la Descentralización y el Desarrollo Regional al afirmar que es "impresentable que dirigentes de algunos partidos presionen para que estas primeras elecciones de intendentes se realicen recién en 2020 o 2021, bajo el pretexto de tener que conocer primero el contenido de las restantes reformas de la agenda de descentralización ${ }^{13}$.Además, manifestó que "la elección de intendentes forma parte de las "medidas esenciales" definidas por la comisión, las que, dada su alta interdependencia, deben ser impulsadas en forma simultánea y convergente".

\section{B. LA AUSENCIA DE MAYORES DEFINICIONES EN PODERES HA- BILITANTES Y AUTONOMISTAS PARA EL INTENDENTE ELECTO}

Von Baer considera de "serias consecuencias" el modelo que define capacidades de gobierno más allá de los temas de seguridad a la figura del gobernador, dando tres argumentos:

1. Significaría abdicar a la oportunidad histórica de iniciar en Chile un proceso de efectiva descentralización (política, administrativa y fiscal-económica), como el que estaba impulsando la Presidenta Bachelet y la Comisión Presidencial, proceso seguido con mucha esperanza por la ciudadanía de las regiones.

2. Crearía una confusa sobreposición de roles entre dos autoridades regionales, muy difícil de entender y de aceptar por la gente en general: Un Intendente Regional "Electo", por ende legitimado por la ciudadanía, pero privado de funciones de gobierno regional, y un Gobernador Regional “Designado, por ende subalterno del poder central.

3. Generaría, inevitablemente, fuertes reacciones en cadena, muy adversas, no sólo de parte de los ex miembros de la Comisión Presidencial (con la consiguiente frustración de haber trabajado seis intensos meses en vano, o incluso, de haber sido designados "para contener y 
no para impulsar este proceso", como se nos señaló en algunos Diálogos Regionales), sino sobre todo de muchas personas e instituciones, a lo largo del país, que habían confiado estar al inicio de un cambio efectivo y creíble del modelo de Estado centralizado vigente ${ }^{14}$.

\section{EL PESO DE LA FIGURA DEL GOBERNADOR REGIONAL}

Por su parte, el profesor de derecho de la U. Austral, Juan Andrés Varas, pone énfasis en el exceso de poder del Gobernador, quien al tener facultades de gobierno tienda a dejar al Intendente electo como un mero administrador del Fondo Nacional para la Desarrollo Regional, FNDR, que además es esencialmente un banco de proyectos municipales para proyectos pequeños y medianos de infraestructura.

El problema de concepción se resume de la siguiente manera: En la clásica división entre funciones de gobierno y funciones de administración, el gobierno regional residirá en el Gobernador Regional (GR) y el Intendente electo se limitará a ejercer la presidencia del Consejo y a administrar lo poco que haya que administrar en la Región (FNDR, y los servicios que el poder central decida unilateralmente traspasar). En esas condiciones, y dado el presidencialismo cultural que existe en Chile, no es muy difícil imaginar quién será considerado "la primera autoridad de la región", y no es muy complicado avizorar los múltiples escenarios de conflicto que se van a abrir entre el GR (apoyado además por su equipo de gobernadores provinciales) y la/el ciudadana/o que resulte electo Intendente. Alguien que tiene el poder pero no la legitimidad democrática directa enfrentado al mismo nivel regional con alguien que tiene esa legitimidad pero ningún (o muy escaso) poder efectivo ${ }^{15}$.

El peso de esta figura delegada encrispó la opinión de los ex comisionados presidenciales, sumándose constitucionalistas de centro como los democratacristianos Alejand ro Ferreiro y Humberto Nogueira, a la crítica a la misma. Finalmente 30 de los 33 ex comisionados firmaron una carta pidiendo a la Presidenta de la República eliminar la figura del gobernador regional, generar un poder que dirima pugnas por competencias (eventualmente el Tribunal Constitucional) y apurar el proceso de traspaso de competencias y

14. Minuta con Observaciones al proyecto de elección de Intendentes. Heinrich Von Baer, diciembre, 2014. p.2 15. Varas, Juan Andrés. Observaciones a proyecto de reforma constitucional del gobierno para la elección de intendentes. enero 2015. pp. 1. 
autonomía fiscal. El 18 de junio marcharon junto a un grupo de parlamentarios regionalistas y directivos de la Asociación Nacional de Consejeros Regionales, ANCORE, hasta el Palacio de La Moneda, siendo la primera ex Comisión nacional que marcha en el país en que sin estar en la calle los sujetos socio-políticos pierden visibilidad y prioridad con sus agendas ${ }^{16}$.

\section{EL MODERADO Y MONITOREADO PROCESO DE TRASPASO DE COMPETENCIAS}

Se ingresó en enero del $2015^{17}$ el proyecto que viene a complementar lo ya avanzado en términos constitucionales en el año 2009 en el primer gobierno de Bachelet, cuando se permitió a los gobiernos regionales en el citado artículo 114 disponer de una excepción al permitir que el Presidente de la República disponga la transferencia de dichas funciones y atribuciones desde un ministerio o un servicio público a uno o más gobiernos regionales.

La propuesta del Gobierno implica dos modalidades de traspaso de competencias, en ambas se requiere la voluntad del Ejecutivo y/o ley:

I. CONVENIO DECRETO SUPREM: Contempla que un Intendente solicite al nivel central, previa aprobación del Consejo, un conjunto de funciones y/o atribuciones que dada su naturaleza para su ejercicio futuro no requiera recursos adicionales, es decir, facultades preferentemente de decisión. Después de la decisión presidencial se suscribirá un convenio entre los niveles central y regional. Esta modalidad se aprueba por Decreto Supremo Ejemplo: El Gobierno Regional decide qué caminos rurales mantener y reparar

II. CONVENIO LEY DE PRESUPUestos: El Gobierno Regional podrá solicitar la administración y ejecución de programas nacionales de claro impacto regional, previo acuerdo con el Ministerio y servicio público respectivo. Esta transferencia se hará efectiva, a través de la incorporación de los recursos tanto para su ejecución y agenciamiento en la Ley de Presupuestos. La naturaleza de este tipo de competencias es esencialmente transitorias. Esta modalidad se aprueba por Decreto

16. Ver "Ex integrantes de la comisión de descentralización exigen apurar agenda a la Moneda" (2015). Disponible en:http://diario.latercera.com/2015/06/19/01/contenido/pais/31-192018-9-ex-integrantes-de-la-comision-dedescentralizacion-exigen-apurar-agenda-a-la.shtml

17. Se formula indicación sustitutiva al proyecto de ley que introduce modificaciones a la ley $n^{\circ} 19.175$, orgánica constitucional sobre gobierno y administración regional, profundizando la regionalización del pais (boletín $\mathrm{N}^{\circ}$ 7963-06). Santiago, 16 de enero de 2015. 
Supremo. Comisiones de servicio de funcionarios no tendrán límite de tiempo Ejemplo: El gobierno regional asigna recursos financieros para la reparación y mantención de caminos rurales ${ }^{18}$.

Este proceso está en las antípodas de lo trabajado por el grupo temático en descentralización administrativa de la Comisión Presidencial, coordinada por el experto José Antonio Abalos, quien desde los inicios del programa de Lagos, junto a la creación del Grupo Los Federales (Valenzuela 2002) propicia el traspaso de servicios claves al gobierno regional autónomo (Abalos 2000) de al menos 20 de los 70 servicios públicos nacionales.

\section{UN ANÁLISIS COMPARADO CON LAS PROPUESTAS DE LA COMISIÓN PRESIDENCIAL Y ALTERNATIVAS}

\section{PERMITIR PODER "REGIONAL DEL INTENDENTE Y ACEPTAR ROLES COORDINADORES NACIONALES DE GOBERNADORES PROVINCIALES}

Si las regiones, los municipios u otros entes territoriales son solamente órganos de administración descentralizados territorialmente, es decir que no tienen Poder político (esto es poder legislativo), se menoscaba simbólicamente lo avanzado en las dos décadas previas. La propuesta del Ejecutivo no propone una regresión, pero al definir en forma amplia los poderes del "gobernador regional" será inevitable las críticas en el Congreso. Es muy probable que desde las fortalecidas bancadas regionalistas del Senado y la Cámara este asunto ocupe un papel clave. Se deben pensar fórmulas políticas que otorguen poder constitucional habilitante al Gobierno Regional y no se deje en el Gobernador Regional tanto las facultades de interior, administración como de gobierno. La crítica en este caso será severísima por generar dos poderes, en vez de delegación-coordinadora y gobierno regional con niveles de autonomía. En este contexto es fundamental dar de manera narrativa explícita el rol de gobierno regional a la Intendencia electa, junto con acotar al gobernador provincial de las capitales regionales un rol coordinador de servicios nacionales. 


\section{FORTALECER EL TRASPASO DE COMPETENCIAS Y TENER INSTANCIA DE NEGOCIACIÓN.}

Habrá críticas evidentes al proceso de traspaso por acotado y largo, dejando regiones sin pilotajes, áreas no tocadas como desarrollo agrícola (INDAP) y programas territoriales de caminos secundarios y manejo de cuencas de Obras Públicas (MOP). Al analizar las reacciones a los proyectos, se puede avizorar que hay críticas por fantasmas. Los centralistas creen que es muy federalista, y los regionalistas que la reforma es mediocre por el extremo poder que puede tener el Gobernador regional y por el traspaso con largos pilotajes que propone la ley.

La Corporación de Fomento Productivo, CORFO fue priorizada por la Comisión Presidencial como institución a ser traspasada a los gobiernos regionales para fomento, innovación y competitividad, además porque puede crear empresas mixtas y programas con la debida flexibilidad. Se ha criticado que CORFO no es proactiva en regiones con rezago (muchas no tienen los programas claves de capital semilla, de riesgo y desarrollo de proveedores locales) y se ha concentrado y centralizado en subsidios financieros a medianas empresas metropolitanas, megaestudios y proyectos asimétricos que se realizan sólo en algunas regiones. Los fondos de fomento regionalizados son sólo el $20 \%$ de su millonario presupuesto. Es posible mantener programas inter-regionales de apoyo a clusters específicos, pero en el mundo desarrollado el dinero de fomento es poder regional para favorecer el desarrollo endógeno de la red de empresas regionales y nuevas oportunidades que requieren sistemas permanentes y no episódicos de apoyo a emprendedores, capitales semillas y otros instrumentos clásicos. El Gobierno propone traspasar sólo programas de CORFO y crear comités con actores privados, pero dejando la agencia centralizada en lo que Carlos Calderón (2014) llama "desconcentrar para no descentralizar".

En un seminario de la U. del Bío Bío, con su Rector Héctor Gaete y el senador Alejandro Navarro, se debatía sobre la urgencia de una alianza público-privado en una suerte de neodesarrollismo sin ortodoxias, para lo cual es clave el poder regional en fomento ${ }^{19}$. Lo mismo ocurre con el ámbito agrícola, donde un Indap fortalecido por la Región, debiera multiplicar sus programas de apoyo, en especial donde se concentra la pobreza rural en el centro norte, el secano de O’Higgins y Maule, y el alto rezago y subinversión en Bío Bío, La Araucanía, Los Ríos y Los Lagos.

El Subsecretario de Desarrollo Regional y Administrativo, Ricardo Cifuentes, enfatiza que "importan más la profundidad que la velocidad de la Reforma 
descentralizadora ${ }^{20 \prime,}$, apelando a que al legitimarse el proceso en algunas regiones podrán venir nuevos servicios y competencias. En dicho sentido es conveniente dejar abierta la puerta para que consejos regionales con su Intendente puedan pedir traspasos- en concordancia con lo que hoy existe en el art. 114 de la Constitución -, y a su vez precisar con mayor fuerza en el texto los poderes de los gobiernos regionales y sus competencias exclusivas para poder reclamar competencias resueltas por el Tribunal Constitucional si en el futuro los gobiernos centrales inhiben el proceso de descentralización que se entiende incremental.

Volviendo al caso de Francia, en su Constitución se precisa en el art. 73, inciso No4 que "Estas reglas no podrán referirse a la nacionalidad, derechos cívicos, garantías de libertades públicas, estado y capacidad de las personas, organización de la justicia, derecho penal, procedimiento penal, política exterior, defensa, seguridad y orden público, moneda, crédito y cambios, así como al derecho electoral.

\section{CREAR LAS ÁREAS METROPOLITANAS Y TRASPASAR TRANSPORTE EN MEGA CIUDADES}

Uno de los artículos del proyecto de fortalecimiento de los gobiernos regionales y traspaso de competencias es la creación de áreas metropolitanas en las zonas con más de 250 mil habitantes, donde el Intendente electo debe gobernar con el Consejo Regional (CORE) y un consejo consultivo de alcaldes involucrados los asuntos de planificación, transporte y disposición final de la basura. Se les dota de una unidad técnico administrativa y se simplifica el modelo como lo propuso la Comisión Presidencial 2014, aunque ésta daba poder de voto a los alcaldes.

El Intendente de la Región Metropolitana (RM) de Santiago, que agrupa treinta municipios, Claudio Orrego, ha vivido la falta de poder y creado una Comisión para pedir competencias y ser pionero en dicho proceso (diciembre 2015). Un sistema sin duplicidades burocráticas que combine la razón tecnocrática y las capacidades profesionales con la corresponsabilidad política en monitorear, destrabar proyectos, asegurar financiamientos sustentables, cobrar lo justo y subvencionar con racionalidad. Orrego no quiere un "zar" del transporte como se suele gestionar el centralismo, más 
bien en su historia contrafactual descentralizadora reclama decisión en el gobierno para darle poder y concentrar competencias, servicios y empresas hoy dispersas. Lo mismo ocurre con la legítima molestia regionalista por la lenta expansión de dicho sistema en Concepción o la ampliación del Metro de Valparaíso (MERVAL) a La Calera. Lo del Metro es evidente. En todos los países desarrollados el transporte está a cargo de la autoridad electa de la región metropolitana o alcalde mayor, como ocurre con la autoridad de transporte de las mejores experiencias del mundo: Tokio, París, Moscú, Londres, Berlín, Nueva York. En este último caso el legislativo regional creó en 1965 el Metropolitan Commuter Transportation Authority, que se fusionó en 1968 con el New York City Transit Authority, la que incluye metros, trenes de cercanía y buses de manera integrada. Desde entonces es clave la MTA Capital Construction Company que lleva a cabo las obras de infraestructuras de toda la red, incluyendo mantenimiento de túneles y puentes. Es de esperar que la ley de traspaso de competencias a los gobiernos regionales otorgue poder en transporte a los intendentes electos. Dicha reforma se ha dialogado con el Consejo Urbano y se espera el pronunciamiento de una Comisión para la movilidad. El propio Ministerio de Transporte ha dicho que tiene claridad en que se debe crear la autoridad metropolitana de transporte, lo que debe expandirse a otras regiones con serios problemas de movilidad en sus zonas de conurbanización donde el Estado no existe, ya que sólo Santiago, Valparaíso y Concepción cuentan con SECTRAs (Secretarias de Coordinación de Transporte), ni equipos técnicos (un "piloto" en Puerto Montt) ${ }^{21}$.

El país tiene enormes déficits de infraestructura de transporte -lo que ha connotado el ex Presidente Lagos- en sus quince áreas metropolitanas, desde las mega (Santiago, Concepción, Valparaíso) a las con urbanizaciones regionales (Iquique-Alto Hospicio, Antofagasta, Coquimbo, la Serena, Rancagua, Machalí, Talca, San Clemente, Chillán, Temuco, Las casas, Puerto Varas, Puerto Montt, o el gran San Antonio, como señalan los estudios de Arturo Orellana (2013).

La siguiente tabla sintetiza el análisis de los proyectos enviados, contrastando las diferencias más relevantes con lo propuesto por la Comisión Presidencial, sugiriendo alternativas de mejoras que rondarán su debate político legislativo. 
TABLA 1

PUNTOS CONTROVERTIBLES DE PROYECTOS DE ELECCIÓN DE INTENDENTES Y TRASPASO DE COMPETENCIAS, CONTRASTE CON COMISIÓN PRESIDENCIAL Y FÓRMULA DE MEJORA

\begin{tabular}{|c|c|c|}
\hline $\begin{array}{l}\text { PROYECTO LEGAL } \\
\text { GOBIERNO }\end{array}$ & PROCESOS DE GESTIÓN & POSIBLE MEJORAS \\
\hline $\begin{array}{l}\text { Elección sin fecha aunque } \\
\text { sugiere } 2017 \\
\text { con nacionales }\end{array}$ & 2016 con Municipales & $\begin{array}{l}\text { Presentar en primer } \\
\text { semestre del } 2015 \\
\text { propuesta de fórmula de } \\
\text { elección, colocar urgencia } \\
\text { y asegurar debate de lo } \\
\text { regional en TV }\end{array}$ \\
\hline $\begin{array}{l}\text { Gobernado regional y } \\
\text { provinciales }\end{array}$ & Delegados provinciales & $\begin{array}{l}\text { Gobernadores } \\
\text { provinciales con roles } \\
\text { de coordinación el de la } \\
\text { capital regional }\end{array}$ \\
\hline $\begin{array}{l}\text { Potestades de Intendente } \\
\text { en lo regional incluye } \\
\text { presidir el CORE }\end{array}$ & $\begin{array}{l}\text { Definición de Estado } \\
\text { regionalizado, } \\
\text { Acompañado de } \\
\text { poderes constituyentes } \\
\text { como GORE, fórmulas } \\
\text { de resolución de } \\
\text { controversia y autonomía }\end{array}$ & $\begin{array}{l}\text { Precisar sus niveles de } \\
\text { autonomía }\end{array}$ \\
\hline $\begin{array}{l}\text { Traspaso de competencias } \\
\text { en base a comité de } \\
\text { ministros y solicitud de } \\
\text { CORE con estudios } \\
\text { Y una década de pilotaje }\end{array}$ & $\begin{array}{l}\text { Paquete básico de } 18 \\
\text { servicios a todos los } \\
\text { gobiernos } \\
\text { Senado media en } \\
\text { caso de discordia por } \\
\text { competencias }\end{array}$ & $\begin{array}{l}\text { Alternativa cuando } \\
\text { un ministerio rechaza } \\
\text { fórmula } \\
\text { Acortar pilotaje de } \\
\text { primer paquete de } \\
\text { servicios } \\
\text { Permitir que GOREs } \\
\text { hagan estudios y } \\
\text { propongan traspasos } \\
\text { desde el } 2015\end{array}$ \\
\hline
\end{tabular}




\begin{tabular}{|c|c|c|}
\hline $\begin{array}{l}\text { PROYECTO LEGAL } \\
\text { GOBIERNO }\end{array}$ & PROCESOS DE GESTIÓN & POSIBLE MEJORAS \\
\hline $\begin{array}{l}\text { Traspaso de servicios, de } \\
\text { partes o nuevos } \\
\text { Foco en SERVIU, } \\
\text { FOSIS, SERNATUR y } \\
\text { parcialmente sistema } \\
\text { CORFO (Sercotec) }\end{array}$ & $\begin{array}{l}\text { Servicios completos o } \\
\text { unidades relevantes } \\
\text { (Vialidad) sin } \\
\text { desmembramiento } \\
\text { parciales } \\
\text { Mejora y } \\
\text { homogenización planta } \\
\text { regional }\end{array}$ & $\begin{array}{l}\text { Dotar al Intendente del } \\
\text { poder de nombrar el } \\
\text { director regional en y } \\
\text { asegurar presupuesto en } \\
\text { base año anterior } \\
\text { Crear figura de } \\
\text { servicios nacionales } \\
\text { regionalizados } \\
\text { Considerar INDAP y } \\
\text { Vialidad (MOP) }\end{array}$ \\
\hline $\begin{array}{l}\text { Crea la divisiones de } \\
\text { fomento/industria, } \\
\text { infraestructura/ } \\
\text { transporte y desarrollo } \\
\text { social }\end{array}$ & $\begin{array}{l}\text { Comisión agregaba } \\
\text { educación/cultura y } \\
\text { nuevo sistema regional } \\
\text { para ciencia y tecnología }\end{array}$ & $\begin{array}{l}\text { Dar rol regional en } \\
\text { ámbitos en nuevo sistema } \\
\text { de administración de } \\
\text { educación pública, que } \\
\text { Intendente nombre en } \\
\text { cultura y sistema mixto } \\
\text { nacional/regional en } \\
\text { CONICYT }\end{array}$ \\
\hline $\begin{array}{l}\text { Crea áreas metropolitana } \\
\text { a cargo del GORE con } \\
\text { un Jefe y tres cargos } \\
\text { profesionales } \\
\text { Comité asesor de } \\
\text { alcaldes }\end{array}$ & $\begin{array}{l}\text { El intendente a cargo } \\
\text { con consejo de alcaldes } \\
\text { que debe aprobar planes } \\
\text { claves, administrador } \\
\text { metropolitano y traspaso } \\
\text { de SECTRAs y servicios/ } \\
\text { empresas de transporte }\end{array}$ & $\begin{array}{l}\text { Poner fechas para las } \\
\text { tres primeras, entregar } \\
\text { el } 2016 \text { a Santiago, } \\
\text { concepción y Valparaíso } \\
\text { poderes en transporte y } \\
\text { vialidad. } \\
\text { Elevar a consejo el } \\
\text { espacio de poder de los } \\
\text { alcaldes }\end{array}$ \\
\hline $\begin{array}{l}\text { Ordenamiento Territorial } \\
\text { es vía decreto que } \\
\text { combina poder sectorial } \\
\text { (ministerios) con opinión } \\
\text { GORE y tras aprobación } \\
\text { es obligatorio }\end{array}$ & $\begin{array}{l}\text { GOREs con poder en el } \\
\text { ordenamiento global } \\
\text { y plebiscitos en temas } \\
\text { controversiales }\end{array}$ & $\begin{array}{l}\text { Acotar los ámbitos en } \\
\text { que los ministerios } \\
\text { cumplen roles de } \\
\text { supervisión de normas } \\
\text { nacionales y potestades } \\
\text { en carreteras energéticas }\end{array}$ \\
\hline
\end{tabular}




\begin{tabular}{|c|c|c|}
\hline $\begin{array}{l}\text { PROYECTO LEGAL } \\
\text { GOBIERNO }\end{array}$ & PROCESOS DE GESTIÓN & POSIBLE MEJORAS \\
\hline $\begin{array}{l}\text { Crea Comité Regional } \\
\text { consultivo público/ } \\
\text { privado en Innovación }\end{array}$ & $\begin{array}{l}\text { Servicio en Ciencia/ } \\
\text { innovación regionalizado } \\
\text { dependiente de GORE } \\
\text { con pluralidad en } \\
\text { consejo }\end{array}$ & Reforma a CONICYT \\
\hline $\begin{array}{l}\text { Poder del CORE: explícita } \\
\text { aprobación de planes, } \\
\text { programas, auditorias } \\
\text { y conocimiento de } \\
\text { Programas Públicos } \\
\text { de Inversiones en la } \\
\text { región (PROPIR), hace } \\
\text { obligatorios convenios de } \\
\text { programación }\end{array}$ & $\begin{array}{l}\text { Rol autónomo de la } \\
\text { región en estos ámbito } \\
\text { y aprobación proyecto a } \\
\text { proyecto en CORE. }\end{array}$ & $\begin{array}{l}\text { Reponer aprobación } \\
\text { proyecto a proyecto } \\
\text { cuando superen las } \\
10 \text { mil UFs } \\
\text { Hacer obligatorio que } \\
\text { CORE aprueba plan anual } \\
\text { de Vialidad, Cuencas, } \\
\text { vías estructurantes, } \\
\text { transporte. }\end{array}$ \\
\hline $\begin{array}{l}\text { Eliminación de número } \\
\text { para identificar regiones }\end{array}$ & $\begin{array}{l}\text { Se propuso que la } \\
\text { Araucanía debatiera su } \\
\text { nombre en su estatuto } \\
\text { de autonomía }\end{array}$ & $\begin{array}{l}\text { Permitir plazo en el } \\
2015 \text { para con consultas } \\
\text { los GOREs por dos } \\
\text { tercios puedan hacer } \\
\text { enmiendas a sus nombres } \\
\text { (Simplificar) o cambiar }\end{array}$ \\
\hline
\end{tabular}

FUENTE: ELABORACIÓN PROPIA EN BASE A LOS PROYECTOS DE LEY INGRESADOS POR EL GOBIERNO EN ENERO DEL AÑO 2015 AL SENADO (REFORMA CONSTITUCIONAL EJECUTIVO REGIONAL) Y LA CÁMARA DE DIPUTADOS (ENMIENDAS SUSTITUTIVAS A PROYECTO DE FORTALECIMIENTO DE LA REGIONALIZACIÓN). ANÁLISIS DEL INFORME DE LA COMISIÓN ASESORA PRESIDENCIAL PARA LA DESCENTRALIZACIÓN Y EL DESARROLLO REGIONAL (OCTUBRE 2014).

\section{C O N C L US I Ó N}

La descentralización en Chile se hace desde la desconfianza y el pragmatismo que implica imponer dicha lógica en coaliciones que no quieren perder poder, con parlamentarios que influyen en los gobiernos delegados y en un contexto económico restrictivo por la desaceleración económica. Para hacer posible el proceso, la fórmula de compromiso en la Nueva Mayoría ha sido acotar los poderes de los gobiernos regionales electos y obligar a traspaso de competencias con voluntad del Ejecutivo central, contratos región-Nación y una serie de sistemas que mantienen el poder institucional, político y fiscal 
en el centro. De este modo, el Segundo Gobierno de Bachelet ha privilegiado focalizarse en descentralización solo en el fortalecimiento del nivel regional con miras a la entrada en vigencia y eventual elección al final de su mandato, con un modelo dual que elige al Intendente, pero da poderes acotados y traspaso de competencias siempre en coordinación con los entes nacionales. Se observa tanto la ausencia de lo municipal en las prioridades (postergado a negociaciones con las asociaciones municipalistas y crispadas por la desmunicipalización de la educación) y a su vez una continuidad con lo ideado en el traspaso de competencia como modelo gradualista e incremental por los equipos de SUBDERE desde el primer gobierno de Bachelet, la administración Piñera y su nueva implementación con Bachelet II.

Con todo, hay un avance de las propuestas reformistas/regionalistas que lograron el envío de un inédito proyecto de elección de intendentes y el compromiso de fortalecer las finanzas autónomas regionales. Al permanecer una fuerte delegación central (gobernadores provinciales, gobernador coordinador, secretarias regionales ministeriales sectoriales y principales servicios), en la práctica se elegirá a un Intendente que se parece a la figura de Presidencia del CORE, aunque las nuevas divisiones regionales (inversión, capital social y fomento) abren la puerta para que con los años se fortalezca la autonomía regional y su mayor capacidad de innovación y de romper brechas de desarrollo.

Se acerca, por tanto, un proceso de poder dual con larga transición hacia un mayor poder de gobierno regional autónomo. Este camino "a la chilena" parece ser el único viable en un país de naturalización del centralismo.

\section{B I B L I O G R A F Í A}

Abalos J. (2000). “Descentralización fiscal y transferencia de competencias: aproximación general y propuestas para fortalecer los gobiernos regionales en Chile". Serie Política Fiscal 102. Santiago: CEPAL/GTZ.

Brosio G. y Jiménez JP. (2012). Decentralization and reform in Latian America. USA: ECLA-GIZ.

Calderón C. (2014). “¿Desconcentrar para no descentralizar?”. Documento de Trabajo Red Desarrollo Territorial (DETE), Noviembre 2014. Disponible en: http://tenred.cl/ wp-content/uploads/2014/11/DESCONCENTRAR-PARA-NO-DESCENTRALIZARCCA-14.11.03.pdf 
Cravacuore D. (2014) Descentralización y recentralización en América Latina (ponencia). XIX Congreso CLAD. Quito.

Debbasch R. (1988), Le principe révolutionnaire d'unité et d'indivisibilité de la République, Paris, Economica, PUAM, 1988.

Delamaza G., Cunill N. y Joignant A. (2012). Nueva agenda de descentralización en Chile. Santiago: Ril-U. de los Lagos.

Delooz B., Pressacco F., y Rivera S. (2014). “Chile Descentralizado. En Valenzuela, Esteban (ed). Aproximaciones a una Nueva Constitución. Santiago El Desconcierto. Pp.36-39.

Falleti T. (2010). Decentralization and Subnational Politics in Latin America. New York: Cambridge University Press.

Horst B. (2007). Modernización del Estado: Descentralización y Fortalecimiento de los Gobiernos Locales. Santiago: Libertad y Desarrollo.

Marimán J. (2012). Autodeterminación. Santiago: LOM Ediciones.

Navarrete B. e Higueras V. (2014). “Chile desde la Teoría secuencial de la descentralización, 1990-2010". Convergencia, núm. 66, septiembre-diciembre 2014, pp. 179-202. México: UAEM.

Orellana A. (2013), “Gobiernos Regionales para Chile”. Documento de Trabajo 63 del Centro de Políticas Públicas de la PUC. Disponible en: http://www.cedeus.cl/ wp-content/uploads/2014/01/N-\%C2\%A6-63-Gobiernos-metropolitanos-para-Chile.La-necesidad-versus-la-factibilidad.pdf

Rosales M. (2012). Descentralización del Estado y Finanzas Municipales en América Latina. Santiago: FLACMA-UB.

Valenzuela E. y Martelli G. (2000). Propuesta para la descentralización y regionalización. Santiago: Fundación Ebert.

Valenzuela E. y Penaglia F. (2014). “Rebeldía en Calama: desafío al orden centralista chileno en un contexto de boom minero. Revista Mexicana de Ciencias Políticas y Sociales UNAM 2014, Vol. 59 (222-250).

Valenzuela E. (1999): Alegato Histórico Regionalista. Santiago: Sur.

Valenzuela E. (2007), “Chile duro: régimen de presidencia centralista reforzada por la dictadura derechista y administrada por la centroizquierda paternalista".En, Carrión, Fernando: Descentralización en Ecuador: opciones comparadas. FLACSO, Quito. 2007pp. 119-142.

Valenzuela E. (2014). "Descentralización: ideal democrático y desarrollo local". En Valenzuela E. y Bello D.: Manual de Ciencia Política. Santiago RIL Editores. Pp.209-234.

Valenzuela E. (2015). Descentralización Ya. Santiago: RIL Editores. 